\title{
Teknokultura
}

ISSNe: $1549-2230$

http://dx.doi.org/10.5209/TEKN.60363

\section{Género, poder y herramientas de comunicación online: relaciones sexoafectivas de pareja en los discursos de jóvenes en Cataluña ${ }^{1}$}

\author{
Priscila Astudillo Mendoza ${ }^{2}$
}

Recibido: 23 de mayo 2018 / Revisado: 26 de junio de 2018 / Revisado: 21 de agosto de 2018 / Aceptado: 12 de septiembre de 2018 Open peer reviews

Resumen. Las herramientas de comunicación online no sólo han ampliado las posibilidades de estrechar lazos sociales con otras personas, sino también parecen estar permeando las relaciones de pareja juveniles en diferentes aspectos, especialmente aquellos vinculados a las performances de género. En este artículo, presentamos los resultados de una investigación cualitativa, fundamentada teóricamente en la noción de poder de Foucault y la performatividad de género de Butler. Utilizamos un análisis de contenido temático desarrollado con una perspectiva feminista, con el objetivo de analizar en los contenidos de los discursos de jóvenes catalanas/es, las relaciones de poder marcadas por género ejercidas en relaciones sexoafectivas de pareja al usar herramientas de comunicación online. Profundizamos en aquellos contenidos que legitimaban y también que desafiaban relaciones de poder patriarcales, principalmente respecto a la clasificación de usuarias/ os según género y con base en ella, la definición de prácticas online de relaciones sexoafectivas de pareja diferenciadas por género. Presentamos los análisis enmarcados en dos categorías: 1) diferencias en función del género y 2) amor y prácticas online de relaciones sexoafectivas. Los principales resultados sugieren la reproducción de discursos patriarcales y también posibilidades concretas para su resistencia. Los discursos que sustentaban éstas últimas emergieron siempre enlazados con discursos hegemónicos individualizantes en un juego constante de reproducción/ transformación.

Palabras clave: discurso individualizante; discursos patriarcal; performatividad de género.

\section{[en] Gender, power and online communication tools: sex-affective couple relationships in the discourse of young people in Catalonia}

\footnotetext{
Abstract. Online communication tools have not only widened the possibility of strengthening social links with others, but they also seem to be permeating different aspects of youth couples relationships, especially those gender-related. In this article, we present the results of a qualitative study, theoretically based on Foucault's concept of power and Butler's gender performativity. We used a thematic content analysis from a feminist perspective framework. The objective is to analyse young Catalonians' discourses and the relationships of power marked by gender of the sex-affective couple, while using online communication tools. We focused on the contents that legitimised and that challenged patriarchal power relations. Mainly regarding the classification of users according to gender, and based on it, the definition of online practices of sex-affective relationships of couples differentiated by gender. We present the analysis framework in two categories: 1) Gender-based differences and 2) Love and online practices in sex-affective relationships. The main results suggest that they reproduce patriarchal

1 Investigación financiada por CONICYT Chile, Programa de Formación de Capital Humano Avanzado, Beca de Doctorado en el Extranjero Becas Chile - Convocatoria 2016, Folio 72170568.

2 Universidad Autónoma de Barcelona (España)

e-mail: priscila.astudillo.mendoza@gmail.com
} 
discourses and also concrete possibilities for its resistance, the discourses that sustained them and how they have always kept linked and emerged with individualizing hegemonic discourses in a constant game of reproduction/transformation.

Keywords: gender performativity; individualizing discourse; patriarchal discourse.

Sumario. 1. Introducción. 2. Nuestros lentes teóricos: género y tecnologías como relaciones de poder. 3. Metodología. 4. Resultados. 5. A modo de conclusión y reflexiones finales. 6. Referencias.

Cómo citar: Astudillo Mendoza, P. (2018). Género, poder y herramientas de comunicación online: relaciones sexoafectivas de pareja en los discursos de jóvenes en Cataluña, en Teknokultura 15(2), 259-274.

\section{Introducción}

Cada vez más aspectos de nuestras vidas dependen, de una u otra forma, de las tecnologías (Wajcman, 2004/2006). Un ejemplo de ello son las plataformas que utilizamos para comunicarnos a través de internet. Estas herramientas se han consolidado en nuestra cotidianidad mediando las relaciones que establecemos con otras personas, en un escenario que parece romper con las barreras tradicionales de la comunicación unidireccional y jerárquica (Yuste, 2015; Ruiz, 2016).

Durante los últimos años el acceso y uso de internet ha aumentado considerablemente. Según las cifras presentadas el 2017 por el Instituto Nacional de Estadística (INE), en España el 83,4\% de los hogares cuenta con acceso a internet y el 84,6\% de la población entre 17 y 74 años la ha utilizado en los últimos tres meses, siendo una práctica mayoritaria entre las/os jóvenes de 16 a 24 años (98\%). En el estudio desarrollado el mismo año por el Interactive Advertising Bureau (2017) el 86\% de las/os internautas declaró utilizar redes sociales (en adelante RR. SS.) para mantenerse en contacto con otras personas.

Las RR. SS. son herramientas de comunicación online que se caracterizan por acoger a una gran cantidad de personas de diferentes lugares del mundo, entre quienes pueden interactuar y comunicarse de manera instantánea, sin mayor esfuerzo y de forma divertida (Ruiz, 2016). Al utilizarlas, sus usuarias/os tienen acceso a distintos niveles de configuración que, como señala Zizi Papacharissi (2009), les permiten redefinir las fronteras geopolíticas en el territorio online; crear límites entre los contenidos públicos/privados y establecer procedimientos para producir y controlar información. Las posibilidades que entregan las RR.SS. están ampliando las formas tradicionales de sociabilidad, donde los vínculos amicales y socioafectivos se abren, proporcionando oportunidades de conocer e interactuar con nuevas/os amigas/os, contactar a personas con las que ya no se mantenía contacto y/o acceder a nuevas parejas potenciales que van más allá de los límites socioespaciales inmediatos (Rodríguez y Rodríguez, 2016).

Para Blanco (2014), las herramientas de comunicación online parecen estar adquiriendo gran relevancia en el desarrollo y mantenimiento de las relaciones sexoafectivas de pareja juveniles. En su estudio desarrollado en España, cerca del 50\% de las/os participantes reconoció haber discutido a causa de publicaciones que involucraban a una tercera persona que no era de confianza o no le agradaba a la pareja. Hombres y mujeres reconocieron controlar y ser controladas/os respecto a quién agregaban o con quién hablaban, identificando un pacto implícito del tipo "tú me controlas a mí, yo te 
controlo a ti" Aun cuando no justificaban la violencia o el control, seguían ejerciéndolos en las RR.SS., muchas veces minimizándolos por considerarlos pruebas de amor, enmarcadas en los discursos hegemónicos del amor romántico.

Ruiz (2016) plantea que, si bien en los usos de este tipo de herramientas se reproducen relaciones asimétricas de género, también estas mismas pueden ser utilizadas para subvertirlas, por ejemplo, mediante la difusión de conocimientos y experiencias que logren sensibilizar sobre la importancia de superar este tipo de relaciones sostenidas en sociedades patriarcales, transmitiendo actitudes igualitarias en diferentes ámbitos como los laborales, la educación o el hogar. Reconociendo la multiplicidad de posibilidades que entregan las tecnologías, las actuales propuestas teóricas feministas están trabajando en una línea de producción mutua entre tecnología y género, en las cuales no caben discursos de plena esperanza o completa negatividad (Reverter, 2013). Como señala Wajcman (2008) aun siendo críticas/os con la ciencia y la tecnología, debemos ser capaces de reconocer su potencial para el ejercicio de nuevas relaciones de poder marcadas por género.

Sensibles al contexto presentado, es en el marco de una investigación feminista más amplia sobre relaciones de poder marcadas por género y usos de tecnologías ${ }^{3}$, en donde nos hemos preguntado cómo en los contenidos de los discursos de jóvenes catalanas/es se estaban reproduciendo y/o desafiando relaciones de poder patriarcales, ejercidas en relaciones sexoafectivas de pareja en los usos de herramientas de comunicación online. Nos referimos a "relaciones de poder patriarcales" para señalar aquellas relaciones asimétricas que, situadas en este momento sociohistórico, organizan las relaciones sociales marcadas por género y también, las maneras en que entendemos dichas relaciones.

Para dar respuesta a la pregunta de investigación y desde una posición feminista, adoptamos una comprensión de las herramientas de comunicación online como tecnologías relacionales en las cuales se performa el género (Gil, Feliu, Rivero y Gil, 2003), e intentamos ser críticas en su análisis, evitando naturalizar la relación joventecnología como si esta fuera inherente a su naturaleza o restringida a determinismos sociales, tecnológicos o modelos homogéneos de desarrollo (Gordo y Megías, 2006). Nos propusimos el objetivo de analizar en los contenidos de los discursos de jóvenes catalanas/es, las relaciones de poder marcadas por género ejercidas en relaciones sexoafectivas de pareja, al usar herramientas de comunicación online, profundizando tanto en aquellos contenidos que legitimaban como en los que desafiaban relaciones de poder patriarcales. Para el desarrollo de este artículo nos centramos principalmente en cómo las/os usuarias/os eran clasificadas/os en categorías diferentes en función del género, y cómo desde ello se definían las prácticas online de relaciones sexoafectivas de pareja.

Buscamos ampliar la mirada sobre las interacciones mutuas que se dan entre las relaciones de poder generizadas y los diferentes usos que parejas juveniles, en sus relaciones sexoafectivas, hacen de las herramientas de comunicación online. Como fin último, pretendemos aportar a la deconstrucción de prácticas discursivas hege-

El trabajo que presentamos es parte de una investigación más amplia, tutorizada por Marisela Montenegro, que comenzó durante el curso del Máster en Investigación e Intervención Psicosocial y ha continuado dentro del Doctorado Persona y Sociedad en el Mundo Contemporáneo, ambos de la Universidad Autónoma de Barcelona. Agradecemos a las/os participantes del estudio, sin sus historias esta investigación no habría sido posible y en especial a todas aquellas amigas y colegas que se dieron el trabajo de nutrir este documento con sus importantes comentarios y aportes. 
mónicas que naturalizan interacciones asimétricas en las relaciones sexoafectivas de pareja, visibilizando y promoviendo aquellos discursos subversivos que logren desestabilizarlas y posibiliten nuevas alternativas de acción.

\section{Nuestros lentes teóricos: género y tecnologías como relaciones de poder}

El enfoque teórico que guió nuestra investigación se fundamentó en la noción de poder desarrollada por Foucault. Sus propuestas rompen con los esquemas tradicionales que conciben el poder como posesión de grupos poderosos, que lo usan desde lo más alto de las estructuras macrosociales. El autor plantea comprenderlo como una práctica relacional con efectos siempre potenciadores, por lo tanto, que debe ser analizado en los espacios microsociales concretos en que se visibilizan sus usos estratégicos, donde se negocian las prácticas de imposición/subversión y en cuyo entramado se legitiman unas u otras verdades (Foucault, 1976/1998).

En complemento con Foucault, nos apoyamos en la teoría de la performatividad del género de Butler (1999/2007), quien plantea que los discursos culturales hegemónicos han configurado el género como un dispositivo de poder. Para la teórica, el género es entendido como una identidad formada débilmente con el tiempo e instaurada mediante la reiteración de actos estilizados del cuerpo. De esta forma, entender el género como performativo alude a su puesta en acto, a entenderlo como una práctica (Butler, 2017).

Si bien son los cuerpos individuales los que actúan las significaciones de género, éstos son evaluados socialmente de manera estricta, con el propósito de mantenerlo dentro del marco binario establecido estratégicamente por los discursos hegemónicos, produciendo y regulando una identificación femenina o masculina asociada a un deseo heterosexual. En consecuencia, una actuación situada fuera de los márgenes establecidos por el sistema sexo-género heteronormativo será castigada, mientras que una buena representación confirmará un supuesto esencialismo a la base, generando la ilusión de un yo con un género constante, necesario y natural (Butler, 1998; 1999/2007).

Estas teorías cobran vida en nuestra investigación, cuando comprendemos que, al usar herramientas de comunicación online, se configuran formas de instrumentalización del poder que también se ejercen en estos espacios cotidianos de interacción microsocial (Constante, 2013). Por lo tanto, consideramos que en estas plataformas de comunicación también se performa el género, las relaciones son actuadas, reproducidas y reinterpretadas por medio del uso que hacemos de ellas (Gil et al., 2003).

Concordamos con Thomas (2005) cuando propone que la política tecnológica tiene mucho en juego en su dimensión performativa, ya que permite la resistencia y la transgresión al abrir espacios para una transformación de las distintas relaciones. Las nuevas tecnologías entregan posibilidades para el desarrollo de nuevas performatividades de categorías prefijadas como el sexo y género y, al mismo tiempo, de la tecnología y su relación con el género (Wajcman, 2010).

Si toda significación tiene lugar dentro de la obligación de repetir, entonces la capacidad de acción está dentro de las opciones de cambiar esta repetición, abriendo la posibilidad de transformar el género, refutando los códigos rígidos de los binarismos jerárquicos y propiciando una subversión de la identidad a través de una práctica de significación repetitiva. La insistencia y proliferación de identidades de género que no se adaptan a las reglas de inteligibilidad cultural, generan grandes oportunidades 
para visibilizar los límites y propósitos que regulan la misma inteligibilidad, posibilitando así también revelar, dentro de los límites de la misma matriz, otras matrices diferentes y subversivas de desorden de género. Por lo tanto, la tarea ya no es saber si hay que repetir, sino cómo repetir y cómo desplazar las mismas reglas de género que permiten la repetición, teniendo en cuenta que no hay posibilidad de repetir fuera de las prácticas discursivas que otorgan la inteligibilidad (Butler, 1999/2007). Al ser capaces de subvertir este tipo de normatividades y abandonar la idea de identidades de género fijas y preexistentes, podemos desarrollar estrategias de análisis y acción política que ayuden a comprender la constitución mutua de las relaciones génerotecnología (Landström, 2007).

\section{Metodología}

Para el cumplimiento de nuestros objetivos de investigación, trabajamos con una metodología cualitativa y utilizamos la herramienta de análisis de contenido temático, el cual, en concordancia con nuestro posicionamiento como investigadoras, lo enfocamos con una perspectiva feminista. El uso de este dispositivo analítico nos permitió realizar interpretaciones a partir del corpus textual, desarrollando lecturas que nos informaron de las condiciones que habían hecho posible su producción y reproducción, pudiendo ir más allá de los datos manifiestos (Vázquez, 1996).

Para seleccionar a las/os participantes definimos cuatro amplios criterios de inclusión: (1) Edad: 18 a 25 años; (2) Diversidad en la composición de género: Mujeres y Hombres (3) Lugar de residencia: Cataluña y (4) Voluntariedad. Por motivos de accesibilidad invitamos a estudiantes de primer año de Psicología de la Universidad Autónoma de Barcelona, curso académico 2015/16, partiendo de la base que ya cumplían con el criterio de edad y en su mayoría vivían en la región.

Desarrollamos 8 entrevistas individuales semiestructuradas, que fueron grabadas luego de obtener el consentimiento de cada participante. Previamente diseñamos un guion flexible cuya estructura general dirigió la conversación. Los principales temas que abordamos incluyeron: (a) descripción y usos generales de herramientas de comunicación online; (b) experiencias positivas y negativas (propias o de terceras personas) en relaciones sexoafectivas de pareja (c) diferencias marcadas por género en el uso de las herramientas, finalmente dejamos un espacio para la reflexión personal acerca del tema. Para asegurar la confidencialidad y también la comodidad de las/os participantes, solicitamos un espacio tranquilo en la universidad para desarrollar las entrevistas.

Al evaluar las posibles consecuencias del proceso de investigación, nos anticipamos a escenarios en los cuales las/os participantes identificaran situaciones de violencia de género en sus relaciones de pareja y fuera indispensable contar con apoyo especializado. Definimos el Centro Joan Rivière en Psicología y Género de la UAB, que ofrece un servicio de acogida y atención psicológica a quienes han vivido o viven violencia de género, como un recurso con el que podíamos asegurar las gestiones y derivaciones que pudieran ser necesarias.

Para el desarrollo del presente artículo, definimos dos grandes categorías analíticas en el marco de los objetivos del estudio. Posteriormente, definimos subcategorías basadas en contenidos más específicos que nutrían las categorías principales y estructuramos el análisis de la siguiente manera: 
1) Diferenciaciones en función del género: a) dicotomía público/privado, b) vigilancia y regulación de la belleza y c) diferencias esencializantes.

2) Practicas online de relaciones sexoafectivas de pareja: a) el chico conquistador/la chica agobiada y b) el control y los celos.

Nos apoyamos en criterios de rigor para garantizar la calidad del estudio. Como señala Vázquez (1996, p. 60), “el material es válido cuando mide lo que está destinado a medir", así los resultados obtenidos deben ser equiparables a los resultados obtenidos por otros analistas usando el mismo procedimiento. En esta investigación resguardamos la validez del estudio invitando a otras investigadoras, también feministas, a revisar nuestros análisis de los datos contrastando sus interpretaciones con las nuestras (Noreña, Alcaraz-Moreno, Rojas y Rebolledo-Malpica, 2012). Además, considerando la agencia de las/os participantes, les presentamos los resultados preliminares del estudio y les invitamos a proponer aquellos cambios que considerasen necesarios. Sin embargo, ninguna/o respondió a nuestra invitación.

\section{Resultados}

\subsection{Diferenciaciones en función del género}

A continuación analizaremos cómo en los contenidos de las entrevistas emergen discursos que definen, diferencian y clasifican a las personas en categorías basadas en los principios del binomio sexo-género. Profundizamos en ellos, ya que nos interesa visibilizar cómo estos discursos van delimitando formas específicas de ser y estar en el mundo y, por lo tanto, también en los espacios online. En este proceso, el género se configura como un dispositivo de poder definido en términos de los discursos culturales hegemónicos, los cuales lo han definido en estructuras binarias (hombre-mujer) y han establecido restricciones enmarcadas en un lenguaje de racionalidad universal que va definiendo sus posibilidades performativas (Butler, 1999/2007).

El primer eje de diferenciación se refiere a la dicotomía entre público y privado. La delimitación de posibilidades performativas en los ámbitos públicos y privados basados en diferencias de género, definen lo que está o no permitido para las chicas en oposición a los chicos y viceversa, en diferentes espacios. Pero ¿cómo se definen estos ámbitos en internet?

Aun cuando las características de cada herramienta permiten definir previamente sus espacios como públicos (perfiles públicos en redes sociales) o privados (mensajería instantánea en WhatsApp), las/os usuarias/os tienen la posibilidad de redefinir los límites, por ejemplo, haciendo público lo privado o privatizando el espacio público al restringirlo a una audiencia seleccionada de contactos. De esta forma, las fronteras se entrecruzan y la interacción va dibujando nuevos espacios mixtos con roles intercambiables, dónde los límites son -o al menos parecen ser- más permeables (Sabater, 2014; Caro, 2015).

El discurso hegemónico heteropatriarcal promueve que las mujeres sean consideradas naturalmente vinculadas a la vida privada y, en consecuencia, poco interesadas o más bien excluidas del ámbito público (Bosch y Ferrer, 2003). Se definen 
así formas correctas de performar la feminidad en una relación sexoafectiva de pareja y, por lo tanto, también en las prácticas desarrolladas en las herramientas de comunicación online:

... era de una foto de ella, que no salía, o sea salía sin ropa pero, no es una foto que digas isales desnuda! [...] o sea se ve su figura, el pelo le tapa el pecho, es una foto que dices jes pa'estar en un museo!. Hizo el cuadro y luego lo puso en Instagram [...] al día siguiente tuvo que quitar la foto, porque el novio como que no podía subir una foto así, que salía desnuda, que como se le ocurría. Y él en cambio, él sí que puede subir fotos sin camiseta, porque claro... es un chico y no pasa nada... y dices a ver... que aquí la culpa también es de la chica de no decir: perdona ¿que no puedo subir una foto cómo? (Participante 1) .

Tal como señala Hubbard (2011), la filosofía ha desarrollado una visión dicotómica en la cual la corporalidad es asociada a la mujer y la mente al hombre, en consecuencia, el cuerpo de una chica es considerado una imagen seductora, no ocurriendo necesariamente lo mismo con el de un chico. El contenido del discurso que emerge en este texto, valiéndose de la ironía como estrategia lingüística, critica las distinciones de género hegemónicas que establecen que un desnudo de mujer no se expone en un perfil público online, mientras que el de un hombre no recibe la misma censura. Esta crítica es capaz de denunciar la norma y ponerla en cuestión.

Entender las TIC como tecnologías relacionales, capaces de condensar y materializar normas y valores sociales, permite comprender cómo en ellas también se realiza el género (Gil-Juárez, Feliu y Vitores, 2010), y, por lo tanto, se configuran como un espacio en los que se definen los límites de lo permitido, donde exhibir un desnudo en una RR. SS. significa hacerlo público. En este fragmento, la exposición de la desnudez de la mujer es reivindicada y respaldada bajo el calificativo de obra de arte en la cual ya ha habido una censura previa del cuerpo (perteneciente a lo privado). Nos preguntamos entonces ¿Qué ocurriría si esta performance no respondiera a criterios artísticos y más bien fuera solo una exposición voluntaria de la desnudez en la red? ¿Existiría aun la misma permisividad?

El contenido analizado es también una crítica a la norma hegemónica que delimita las formas aceptadas de performar el género en relaciones sexoafectivas heterosexuales de parejas. El control online pareciera estar facilitado por una suerte de panoptismo que favorecería la vigilancia a las actividades del/la otro/a, promoviendo la corrección de las performances situadas fuera de la norma, para traerlas nuevamente al terreno de lo normal (Constante, 2013). En este ejemplo, dotando al chico del poder de censurar y controlar a la chica frente a una performance que se aleja de lo esperado para su género, siendo ésta quien se somete a la imposición, ejerciéndose una relación de poder en términos de dominio/subordinación entre hombre/mujer.

Aun cuando la crítica se dirige al contenido patriarcal en este tipo de interacciones y es capaz de enfrentarla, finalmente la responsabilidad de permitir este tipo de relaciones asimétricas recae individualmente en la chica, al no cuestionar la situación ni hacerle frente al novio, invisibilizando los motivos de género que están a la base. Pese a ello, consideramos que el poner en cuestión la norma y

4 Hemos respetado en lo posible una transcripción literal de las declaraciones originales de las/os participantes. 
enfrentar el control ejercido, es una posibilidad concreta de subvertir la relación de poder al identificar la desigualdad entre chicos y chicas en este tipo de performance. Las herramientas de Comunicación online, al entrecruzar los límites de público/privado y hacerlos más permeables (Papacharissi, 2009; Sabater, 2014), permiten abrir los espacios de interacción íntima a otras audiencias invisibles, favoreciendo un mayor control de la situación social (Caro, 2015), pudiendo generar nuevos espacios para el enfrentamiento de este tipo de relaciones de poder asimétricas.

Otro punto a analizar es cómo este nuevo tipo de panóptico presente en las RR. SS., promueve la vigilancia de las performances en los espacios públicos online (Constante, 2013), con el propósito estratégico de mantener el género dentro del marco binario establecido por los discursos hegemónicos (Butler, 1999/2007). Un ejemplo de ello es la imposición y regulación de la belleza como mandato de género para las mujeres.

... por ejemplo vas a la universidad el primer día de clases y ves alguna foto en Facebook de un grupo y dices ¡halá, mira esa chica será muy guapa, que seguro todos los chicos le andan detrás! (Participante 3).

La vigilancia de la belleza de otras usuarias reproduce un discurso que promueve relaciones de poder asimétricas marcadas por género, respecto a la sexualización de las mujeres y, con ello, la subordinación en cuanto a objeto de deseo para los chicos. Como señala Naomi Wolf (1992), el mito de la belleza propone un presunto modelo universal y objetivo, al cual las mujeres deben aspirar a personificar y los hombres a poseer.

Muchas chicas yo creo que... yo me incluyo, las utilizamos [las RR.SS.] como... nuestro espejo al público [...] las chicas creo que mucho más cuidan el tipo de fotos que suben, el aspecto de esas fotos... lo bonita que son, los efectos, no sé, no sé cuánto, en las redes sociales tipo Instagram y Facebook, que los chicos (Participante 3).

En los espacios públicos de interacción presentes en las RR.SS., están delimitadas también las posibilidades performativas a las cuales sus usuarias/os se ven interpeladas a personificar. Como plantea Judith Butler (1999/2007), la norma que concibe al sexo como imperativo, interpela al sujeto y va produciendo y regulando una identificación femenina o masculina. Esta identificación nunca logra ser completamente exhaustiva, ni logra interiorizarse del todo, generando constantes críticas a una/o misma/o y llevando a exagerar las exhibiciones públicas de lo natural ("cuidan el tipo de fotos que suben" "lo bonita que son" "los efectos"), mostrando su carácter fantasmático que lo vuelve imposible de personificar tal y como es interpelado.

El ejercicio de esta vigilancia constante es puesto en evidencia e identificado como comportamiento normalizado entre chicas:

puede ser que a lo mejor las chicas sean más... sea más para cotillar, o sea más para ver qué hacen los demás y los chicos creo que no tanto (Participante 7). 
La redefinición de las fronteras entre lo público y lo privado y, la posibilidad de acceder atemporalmente a las publicaciones de otras/os usuarias/os, son características de las herramientas de comunicación online que parecen facilitar el proceso de vigilancia-regulación del que venimos hablando. Al delegar estratégicamente en las chicas, y no a los chicos, la responsabilidad de "cotillear", de "ver qué hacen los demás", el discurso hegemónico heteropatriarcal refuerza la normalización de esta performance y la sitúa en el terreno de la esencia, de lo naturalmente femenino "las chicas sean más", encubriendo la estrategia reguladora que hay detrás de ella.

Consideramos que este tipo de relaciones de vigilancia-regulación de performances entre chicas, donde la belleza es el imperativo y el cuidado de las exposiciones públicas cobra un rol transversal, refuerza la reproducción de relaciones patriarcales, incluso entre las propias chicas, al cosificar sus cuerpos como objetos de deseo al servicio del patriarcado.

Profundizaremos también en cómo desde un uso específico del lenguaje por medio de deixis como "las mujeres", "la chica", "el novio" o "los hombres", el discurso refiere a las personas identificándolas y clasificándolas en categorías de género preestablecidas y homogéneas. Con base en ello, las explicaciones respecto a las diferencias en las performances de género online, es decir, cómo chicos y chicas actúan en determinadas situaciones al usar estas herramientas de comunicación, se basan principalmente en argumentos que buscan confirmar una supuesta naturalidad a la base:

...las chicas somos como más... nos gusta más expresar nuestros sentimientos, hablar de cómo nos sentimos y... cosas parecidas, entonces como que cuando nos sentimos de una manera, en un momento concreto, de seguida lo estamos explicando por WhatsApp con nuestras amigas o a nuestros novios y ellos como que... cuando sienten algo muy fuerte o cuesta mucho que te lo digan por WhatsApp o te lo dicen en persona, pero si, yo creo que nosotras somos más así de soltarlo y a quien sea, en el momento que sea, entonces por WhatsApp, por teléfono como sea, lo hacemos enseguida... (Participante 2).

En la dicotomía hombre/mujer se reproduce la oposición cultura/naturaleza, vinculando lo femenino a elementos naturalizantes y esencializantes como lo es, por ejemplo, la emocionalidad (Amigot y Pujal, 2009). La identificación con una categoría generalizada "nosotras" en contraposición con "ellos", promueve explicaciones esencialistas del tipo "las chicas somos", "nos sentimos", "nos gusta más expresar nuestros sentimientos", reforzando un imaginario de mujeres mucho más emocionales y con la necesidad de mantener el contacto con otras personas, invisibilizando las diferencias al interior de cada grupo y definiendo una forma específica de ser y estar en el mundo. Estos atributos distintivos del género no son casuales, sino que van contribuyendo a la humanización de los individuos al interior de la cultura contemporánea, donde una buena representación confirmará el supuesto esencialismo a la base de la identidad de género (Butler, 1998). Así, discursivamente se valida la expresión emocional de las mujeres en el ámbito público y también de manera privada, a diferencia de lo que analizamos anteriormente respecto a la desnudez de la mujer, la cual no está permitida en espacios públicos.

Por el contrario, respecto a los chicos, la expresividad emocional en público parece ser negada. Para "ellos", asociados a la racionalidad, esta performance está 
permitida exclusivamente en la intimidad y sólo cuando "sienten algo muy fuerte". A diferencia de las chicas, el uso de la herramienta de comunicación online no representa un soporte privilegiado para que los chicos comuniquen la emoción, prefiriendo el contacto offline ("te lo dicen en persona").

Las explicaciones esencialistas sobre las diferencias de género, promueven el mantenimiento de relaciones de poder patriarcales, ya que al reconocerlas como características naturales estables y propias de un grupo específico, reducen las posibilidades de proponer nuevas performances alternativas capaces de subvertirlas. Por otro lado, consideramos valiosas las posibilidades que entregan estas herramientas para poder hacer de vehículo comunicativo, que permiten buscar soporte emocional (por lo menos a las chicas) con otras personas de forma inmediata sobrepasando las barreras físicas.

\subsection{Amor y prácticas online de relaciones sexoafectivas}

Profundizaremos en cómo los discursos hegemónicos patriarcales delegan estratégicamente a las herramientas de comunicación online la capacidad en sí mismas de generar, favorecer o promover ciertos tipos de interacciones, con base en ello se sostienen prácticas y desarrollan explicaciones respecto a las formas posibles de performar las relaciones sexoafectivas de pareja enmarcadas en los términos propios de estos discursos. A pesar de ello, identificamos también opciones concretas para hacer frente a este tipo de relaciones patriarcales.

El uso de herramientas de comunicación online ha ampliado las posibilidades de conocer nuevas parejas potenciales, implicando incluso menores costos económicos y emocionales que de manera offline o "en persona". La flexibilidad de los límites entre lo público y privado, que hemos estado analizando, permite a las/os usuarias/ os acceder a la información personal de otra persona en los perfiles públicos de las RR.SS., incluso antes de decidirse a iniciar algún tipo de contacto, permitiendo emplear estrategias de conquista ante un mayor número de personas, de manera selectiva, rápida y fácil (Rodríguez y Rodríguez, 2015).

Una vez comenté en un post [...] y un chico le dio me gusta y me agregó, me hablaba todos los días!... y yo pensaba que pesado que es y claro, tampoco yo puedo ir como ohhh quiere ligar conmigo, porque eso es ir de subido, entonces tienes que ser una persona cordial y lo primero que me pregunta claro: anda he visto tu post [...] entonces debatimos un poco sobre el tema y pensé: bueno ya está. Y luego me siguió hablando, me siguió preguntando, quería hablar conmigo todos los días y yo: es muy pesado y al final dije: mira te tengo que bloquear [...] lo bloqueé y dije ¡ah, ya está! (Participante 1).

... primero lo intentas de plan... bueno si ya hablaremos mañana ¿sabes? pero el chico no ha dejado de hablarle al punto de que ella ha tenido que... [bloquearlo], porque no, no, hay barreras que no se pueden pasar [...] si ya el problema lo tiene la persona sin haber comunicación por internet, con la comunicación por internet todavía peor... (Participante 5).

Las prácticas románticas de relaciones sexoafectivas de pareja, enmarcadas en discurso hegemónico heteropatriarcal, suponen la subordinación y objetivación de lo femenino frente a lo masculino, donde la mujer es desprovista de su capacidad de 
agencia a la espera de ser "conquistada" (Sánchez-Sicilia y Cubells, 2018). De esta forma, es el chico quien debe cumplir el mandato de género y emplear activamente estrategias de cortejo y mantenerlas aun cuando está siendo rechazado. En complemento, se delega en las mujeres la responsabilidad de establecer límites en estas situaciones, donde el malestar de una chica, en primera instancia, no puede ser expresado directamente, sino que debe performarse sutilmente dentro de los márgenes definidos para la feminidad. Se utilizan estrategias lingüísticas ("ya hablaremos mañana") como una manera indirecta para cortar la relación, respondiendo a la pasividad femenina desde la cual deben ser enfrentadas estas situaciones. Esta interacción refuerza además, el actuar del chico como un conquistador que no responde a formas sutiles de alejamiento ("me siguió hablando, me siguió preguntando", "el chico no dejó de hablarle"), conformándose un espiral que va repitiendo esta relación asimétrica y que se refuerza con la comprensión de las RR. SS. como herramientas que por sí mismas exacerban este tipo de problemas también presentes en las relaciones offline, reproduciendo así el ejercicio de relaciones de poder patriarcales.

Destacamos como emerge también, un discurso capaz de identificar y resistir la relación de poder analizada, al reconocer que las herramientas de comunicación online posibilitan una agencia activa por parte de las chicas permitiéndoles cortar el contacto cuando así lo requieran. Consideramos que esta posibilidad de terminar el contacto no logra subvertir el discurso hegemónico que define las maneras de performar el género, más bien encubre el mantenimiento de relaciones de poder asimétricas desfavoreciendo a las mujeres, quienes se ven obligadas a poner los límites a un "conquistador" que no responde a formas sutiles de alejamiento.

Otro punto relevante que analizaremos es cómo las RR.SS. online, al mantener un registro histórico de las publicaciones de sus usuarias/os, tales como pensamientos, emociones y vivencias, parecen flexibilizar las barreras del tiempo y espacio al permitir acceder de manera asincrónica a las publicaciones de otra persona. Ahora bien, como señalan Rodríguez y Rodríguez (2016) estos registros corren el riesgo de ser utilizados para ejercer el control, por medio de la vigilancia y monitoreo constante.

...las redes sociales sí que tienen algo negativo, es, el hecho de, ejercer el control, te permite ejercer más control aún sobre la otra pareja solo por el hecho de que le has dado me gusta a una foto, o te he visto hablar, o he visto un mensaje, que hace esta persona o te deja un comentario, eso genera problemas cuando él, la otra persona es insegura, en ese caso sí que es algo negativo en las relaciones, y por eso permite ejercer más control sobre una persona porque puedes controlar todos sus movimientos. Al fin y al cabo, en Facebook, tú puedes ver todo lo que hace esa persona; claro y a tu pareja no se los vas a ocultar [...] claro, entonces, es eso, te permite ejercer control, sea una persona de un género u otro, da igual (Participante 4).

...ahora también veo de control [...] mucho también por parte de las mujeres, que y por igual en hombres y mujeres de controlar por WhatsApp, ehhh dónde estás, qué estás haciendo, esto... bueno esto pasa [...] es algo malo que veo [...] al final no sé qué acaba haciendo que nos comportemos así, la verdad (Participante 8). 
Las RR.SS. online parecen ampliar las posibilidades de usar estrategias para controlar a la pareja, "ya sea por parte de chicos o de chicas" "por el hecho de que le has dado me gusta a una foto, o te he visto hablar, o he visto un mensaje". Para Cristina Cabedo (2013), el ejercicio del control a la pareja en estos entornos de comunicación es validado bajo la idea de propiedad difundido por los postulados del amor romántico "a tu pareja no se los vas a ocultar".

La redefinición de los antiguos contornos del tiempo y el espacio, así como la permeabilidad de lo público y privado (Papacharissi, 2009; Sabater, 2014) permiten a las/os usuarias/os y a sus parejas acceder a los registros de publicaciones (ahora públicos o semipúblicos) incluso de manera asincrónica ("ver todo lo que hace esa persona"), reforzando una ilusión de pasividad e incluso de obligatoriedad frente a estas performances ("al final no sé qué acaba haciendo que nos comportemos así").

El ejercicio del control parece ser un problema de pareja exclusivamente cuando las personas necesitan hacerlo debido a sus características individuales, cuando "la otra persona es insegura, en ese caso sí que es algo negativo en las relaciones", legitimando desde la psicologización el ejercicio del poder y dotando a las herramientas de comunicación online de una suerte de conductora y facilitadora del control. Se digitalizan así, relaciones de poder asimétricas, que se pueden hacer presentes las 24 horas al día y que muchas veces pasan desapercibidas al normalizarlas como pruebas de amor (Blanco, 2014).

en el tema de redes sociales, pues terceras personas que te agregan [...] y a veces, pues hay personas que te vienen a tocar la fibra ¿no? y... bueno pues... tener mala suerte, pero no sienta bien a una pareja que haya otra persona ahí, pues hola guapa, que guapa estas ¿no? y sí, me ha pasado también un millón de veces [...] yo supongo que depende si tu novio es más celoso o menos celoso también (Participante 6).

El discurso del amor romántico respaldado en el mito de la exclusividad (Blanco, 2014), valida los celos frente a una posible infidelidad ("no sienta bien a una pareja que haya otra persona ahí"), situada principalmente en los márgenes del sistema sexo-género heteronormativo. La flexibilización de los límites público/ privado en las herramientas de comunicación online y la aparente pasividad con la que se hace uso de las posibilidades concretas para acceder de manera asincrónica a las publicaciones de la pareja, parecen naturalizar el ejercicio de control y la expresividad de los celos, pudiendo incluso justificarlos, respaldándose en los mitos la posesividad y exclusividad enmarcados en el discurso del amor romántico. Se justifica entonces, la expresión de los celos que emergen en un contexto que, facilitado por las herramientas de comunicación online, parece respaldarlos, como al existir "terceras personas que te agregan" a la red de contactos o en presencia de "personas que te viene a tocar la fibra", recurriendo a características individualizantes y psicologizantes para justificarlos.

Identificamos también discursos capaces de enfrentar e incluso desestabilizar estas relaciones de poder analizadas:

... el día que me diga: ay!! he visto que has comentado esto ¿por qué? y yo le diré: Perdona no, o sea ¡no!. Hay que poner límites. Tener una red social para comunicarte o usar un WhatsApp o lo que sea, no puede ser, o sea no es sinónimo de mi 
pareja ya no tiene privacidad, [...] está muy bien ser pareja, pero no significa que tú pasas a ser vosotros, tú eres tú y aparte después sois un vosotros ¿no?, pero eso no implica: ay! se ha desconectado del WhatsApp dos horas después de decirme que se desconectaba, porque a mí el día que me pregunte eso diré: no, perdona ya me quitó la última conexión (Participante 1).

Este desafío al discurso hegemónico se desarrolla desde otra posición discursiva hegemónica: la individualista, en la que el "yo" es puesto en el centro de las decisiones y de la vida. La crítica y enfrentamiento directo al ejercicio del control en el uso de estas herramientas de comunicación, se fundamenta en la individualidad de la otra persona, la que podría ser vulnerada en la flexibilización de los límites entre lo público y lo privado "no es sinónimo de mi pareja ya no tiene privacidad". Este enfrentamiento, es capaz de desafiar las relaciones de poder a la base, reconociendo posibilidades de agencia activa para las/os usuarias/os, tanto en la definición de límites en la pareja, como en la oportunidad de manejar las opciones de configuración de la propia herramienta de comunicación "perdona ya me quitó la última conexión”, pudiendo definir así la información que quiere compartir y la que no.

\section{A modo de conclusión y reflexiones finales}

La adopción de una perspectiva foucaultiana respecto al poder, permitió reconocer como en las herramientas de comunicación online, consideradas espacios microsociales de interacción, el ejercicio del poder se ejercía en las relaciones cotidianas entre las/os usuaria/os y sus parejas. Pudimos observar que tanto la propia herramienta tecnológica como sus prácticas de apropiación eran performativas de género, performances que fueron reproducidas y reinterpretadas por medio de los diferentes usos que las/os jóvenes hacían de ellas (Remondino, 2012; Gil et al., 2003).

En cuanto a la reproducción de discursos hegemónicos, el principal es aquel que promueve la coherencia con el sistema sexo-género heteronormativo (Butler, 1998; 1999/2007), definiendo las formas correctas de ser/estar en una relación sexoafectiva y también de performar el género, sobre todo en un contexto en el que las herramientas de comunicación online se configuran como tecnologías relacionales (Gil et al., 2003) capaces de permear las interacciones sexoafectivas y sostener prácticas androcéntricas (Blanco, 2014), como lo son los roles definidos para el cortejo, el ejercicio del control e incluso la expresión de los celos, respaldados por los mitos del amor romántico.

Los mecanismos de vigilancia y control presentes en estos entornos de relación delegan estratégicamente a las herramientas de comunicación online la responsabilidad de permitir, facilitar e incluso parecer obligar a sus usuarias/os a ejercer éstas relaciones de poder patriarcales, invisibilizando los motivos de género que están a la base, pudiendo incluso a llegar a ejercer y justificar la violencia de género.

Basándonos en los contenidos analizados en el presente artículo, emerge un discurso capaz de reconocer en las opciones de configuración de las propias herramientas posibilidades concretas de enfrentar relaciones de poder asimétricas, dotando a las/os usuarias/os de una agencia activa para (a) flexibilizar los límites espaciotemporales que regulan la información que está o no disponible y quién o quiénes tendrán acceso a ella, (b) contar con un vehículo comunicativo inmediato que les 
permita solicitar soporte emocional o ayuda frente a algún problema y (c) cortar el contacto con alguien cuando la/el usuaria/o lo estime conveniente.

Si bien las posibilidades performativas diferenciadas para hombres y mujeres siguen estando presentes, las fronteras entre lo público y lo privado son más difusas, permitiendo a sus usuarias/os limitar o ampliar las audiencias frente a prácticas específicas. Consideramos que estas posibilidades de hacer público o al menos semipúblico lo que en otro contexto habría sido privado, posibilita la participación de nuevas audiencias, capaces de desarrollar una mayor evaluación social de las performances de género, pudiendo problematizar y enfrentar el ejercicio de relaciones de poder patriarcales ejercidas en estos espacios microsociales de interacción.

Destacamos también como los discursos capaces de desestabilizar e incluso subvertir las relaciones de poder patriarcales en estos entornos relacionales, emergieron siempre enlazados con discursos hegemónicos que tenían efectos de individualización, recurriendo por ejemplo a explicaciones psicologizantes para respaldar los celos, a interpretaciones esencializantes para dar sentido a las diferencias entre chicos y chicas, e incluso al individualismo como motivo para respetar a la otra persona. Estos discursos se nutrían y potenciaban reiteradamente, en un juego de transformación y reproducción de relaciones de poder asimétricas marcadas por género.

Finalmente, aun cuando identificamos posibilidades concretas de enfrentamiento al discurso hegemónico, consideramos necesario seguir problematizando cómo las relaciones entre jóvenes y tecnología se vuelven espacios de interacción obvia, en los cuales las performances de género se naturalizan y las diferencias en el ejercicio del poder se explican desde una supuesta esencialidad a la base (Gil-Juárez et al., 2010), que al ser reconocida como una característica estable y propia de un grupo específico, reduce las posibilidades de proponer nuevas performances alternativas capaces de subvertirlas.

\section{Referencias}

Amigot, P. y Pujal, M. (2009). Una lectura del género como dispositivo de poder. Sociológica, 70, 115-152.

Blanco, M. A. (2014). Implicaciones del uso de las redes sociales en el aumento de la violencia de género en adolescentes. Comunicación y Medios, 30, 124-141.

Bosch, E. y Ferrer, V. (2003). Fragilidad y debilidad como elementos fundamentales del estereotipo tradicional femenino. Feminismo/s, 2, 139-152. doi: 10.14198/fem.2003.2.09.

Butler, J. (1998). Actos performativos y constitución del género: un ensayo sobre fenomenología y teoría feminista. Debate Feminista, 18, 296-314.

Butler, J. (1999/2007). El género en disputa. Barcelona, España: Paidós Ibérica.

Butler, J. (2017). Cuerpos aliados y lucha política. Barcelona, España: Paidós.

Cabedo, C. (Noviembre de 2013). El falso empoderamiento de la mujer como detonador de la Violencia de Género en una cultura Pop, Cibernética y Romántica: la Sextorsión. En Directora General de Violencia de Género y Asistencia a Víctimas de la Consejería de Justicia e Interior de la Junta de Andalucía (Presidencia), IV Congreso para el estudio de la Violencia sobre las Mujeres. Sevilla, España.

Caro, L. (2015). WhatsApp y la búsqueda de control de la situación informacional por parte de los jóvenes. Sphera Pública, 15, 76-96. 
Constante, A. (2013). ¿Qué son las redes sociales? En A. Constante (Coord.), Las redes sociales una manera de pensar el mundo (pp. 13-36). DF, México: Ediciones Sin Nombre S.A. de C.V.

Foucault, M. (1976/1998). Historia de la sexualidad I: la voluntad de saber. Madrid, España: Siglo XXI Editores.

Gil, A., Feliu, J., Rivero, I. y Gil, E. (2003). ¿Nuevas tecnologías de la información y la comunicación o nuevas tecnologías de relación? Niños, jóvenes y cultura digital [en línea]. Disponible en: http://www.uoc.edu/dt/20347/index.html

Gil-Juárez, A., Feliu, J. y Vitores, A. (2010). Performatividad tecnológica de género: explorando la brecha digital en el mundo del videojuego. Quaderns de Psicología, 12(2), 209-226.

Gordo, A. y Megías, I. (2006). Jóvenes y cultura Messenger: tecnologías de la información y la comunicación en la sociedad interactiva. Madrid, España: FAD - INJUVE.

Hubbard, K. (2011). Sexo asimétrico: el pensamiento no dicotómico del cuerpo a partir de la sexualización del otro (sobre algunas fotos de María Zorzón y Gabriela Liffschitz). (Tesis de Master) Misisipi, EE. UU.: Louisiana State University and Agricultural and Mechanical College.

Instituto Nacional de Estadística [INE]. (2017). Encuesta sobre equipamiento y uso de tecnologías de información y comunicación en los hogares. Año 2017. Disponible en: http://www.ine.es/prensa/tich_2017.pdf

Interactive Advertising Bureau [IAB Spain]. (2017). Estudio anual redes sociales 2017 [en línea]. Disponible en: https://iabspain.es/wp-content/uploads/iab_estudioredessociales_2017_ vreducida.pdf.

Landström, C. (2007). Queering feminist technology studies. Feminist Theory, 8(1), 7-26.

Noreña, A., Alcaraz-Moreno, N., Rojas, J. y Rebolledo-Malpica, D. (2012). Aplicabilidad de los criterios de rigor y éticos en la investigación cualitativa. Aquichan, 12(3), 263-274.

Papacharissi, Z. (2009). The virtual geographies of social networks: a comparative analysis of Facebook, LinkedIn and ASmallWorld. New media \& Society, 11(1\&2), 199-220.

Remondino, G. (2012). Blog y redes sociales: un análisis desde las tecnologías de la gubernamentalidad y el género. Athenea Digital, 12(3), 51-69.

Reverter, S. (2013). Ciberfeminismo: de virtual a político. Teknokultura, 10(2), 451-461. Disponible en http://revistas.ucm.es/index.php/TEKN/article/view/51905/48039

Rodríguez, T. y Rodríguez, Z. (2016). El amor y las nuevas tecnologías: experiencias de comunicación y conflicto. Comunicación y Sociedad, 25, 15-41.

Ruiz, M. (2016). Sexismo en línea: WhatsApp, nuevo mecanismo de reproducción del sexismo. Jaén, España: Diputación Provincial de Jaén - Igualdad y Bienestar Social.

Sabater, C. (2014). La vida privada en la sociedad digital. La exposición pública de los jóvenes en internet. Aposta Revista de Ciencias Sociales, 61.

Sánchez-Sicilia, A. y Cubells, J. (2018). Amor, posmodernidad y perspectiva de género: entre el amor romántico y el amor líquido. Investigaciones feministas, 9(1), 151-171.

Thomas, D. (2005). Hacking the body: code, performance and corporeality. New Media \& Society, 7, 647-662. doi:10.1177/1461444805056010.

Vázquez, F. (1996). El análisis de contenido temático. Objetivos y medios en la investigación psicosocial. (Documento de trabajo). (pp. 47-70). Universitat Autónoma de Barcelona.

Wajcman, J. (2004/2006). El tecnofeminismo. Madrid, España: Ediciones Cátedra.

Wajcman, J. (2008). Continuidad y cambio. Género y culturas de la tecnología en el trabajo. Telos: Cuadernos de comunicación e innovación, 74.

Wajcman, J. (2010). Feminist theories of technology. Cambridge Journal of Economics, 34(1), 143-152. doi:10.1093/cje/ben057. 
Wolf, N. (1992). El mito de la belleza. (Cristina Reynoso, trad.) Debate Feminista, 3(5). 214-224.

Yuste, B. (2015). Las nuevas formas de consumir información de los jóvenes. Jóvenes y generación 2020, 108, 179-191. 